\title{
The outsourcing and commercialization of science
}

\author{
Is the lab CEO the future of academic research?
}

\author{
David R Smith
}

( nce upon a time, there was a small academic research laboratory, in an old brick building, on a quaint college campus. Though small, the lab had everything it needed to do quality research and publish in high-impact journals. There were a handful of keen undergraduate volunteers, a couple of energetic and optimistic grad students, and an old, silverback PI with a modest but sufficiently sized grant. If you peeked through the ivy-covered windows of this small lab, you would likely spot some large pieces of equipment, like a fridge, fume hood, centrifuge, and computers, and a cluttering of microscopes, beakers, test tubes, and chemical jars. Occasionally, a student would need an instrument or a reagent that was not in the lab. When this happened, the PI grunted and guffawed and then bought the missing item or sent the student down the hall to borrow it from another team. But apart from that, the lab was self-sufficient and produced a steady stream of papers.

This depiction of autonomous research might sound foreign and outdated to many scientists reading this article, but it was once the norm across most universities-just ask that friendly professor emeritus who putters up and down your department hallways. Sure, contemporary research labs are still populated by ambitious students and grizzled PIs; they are still littered with high-tech tools, flashy computers, and expensive chemicals; and they still try to publish in "big” journals. But in today's fast-paced academic landscape, producing quality research is no longer a solely in-house endeavor; rather, it is increasingly dependent on collaborations, alliances, and outsourcing [1]. The days of the lone-wolf researcher are long gone, and nowhere is this more apparent than in the field of genomics.

A nyone who has spent time sequencing or analyzing genomes in the past decade can attest that much of the work is farmed out to private companies. For example, in a number of labs, the species being studied are collected, grown, and maintained by private or public culture collections, such as the American Type Culture Collection (ATCC) or the National Center for Marine Algae and Microbiota (NCMA). In recent years, culture collections have started going a lot further than just providing strains to researchers. For a moderate fee, they will not only grow the organism for you, but also isolate and purify its DNA, and perform a range of other experiments. The ATCC website articulates this point well: "If you can't find the nucleic acids that you need from [the] catalog ... ask ATCC to make it for you! Small-scale quantities of nucleic acids from ATCC Genuine Cultures are ideal for PCR, cloning, or other molecular applications. If your research requires larger quantities of nucleic acids, we can grow, extract and purify [them] from most ATCC cultures" (www.atcc.org).

And we all know the sad, glorious fate that awaits most of these purified DNAs: straight to the commercial sequencing center chopping block [2]. The ability and infrastructure to do in-lab molecular sequencing was once the hallmark of a successful research group, but nowadays sequencing is almost always outsourced and seldom performed by the people that actually publish the data [3]. In the late 1990s and early 2000s, when commercial sequencing started taking off, researchers typically shipped off their PCR products and clones to be sequenced on old-school Sanger machines [4]. Now, major sequencing companies, such as Macrogen (www.macrogen.com), Source BioScience (www.lifesciences.sourcebioscience.com), and GE Healthcare's SeqWright (www.seqwright. com), offer everything from whole genome and transcriptome sequencing on a range of next-generation platforms to expression analysis, genotyping, and variant detection to epigenomic profiling, chromatin immunopreciptation sequencing (ChIP-Seq), and proteomics-and, yes, they still sequence good ol' PCR products. In some instances, customers do not need to provide highquality DNA or RNA; the provider will isolate it for them, design the libraries or primers, and even assemble the reads.

“... in today's fast-paced academic landscape, producing quality research is no longer a solely in-house endeavor ..."

It does not end there. These same companies can even store your samples. Source BioScience and SeqWright, for instance, provide an array of biorepository services, including specialized simulated environments, 24/7 tracking and monitoring of the samples, hazardous specimen storage capabilities, and emergency backup systems. If biotech companies are housing your samples and generating your data, you might as well 


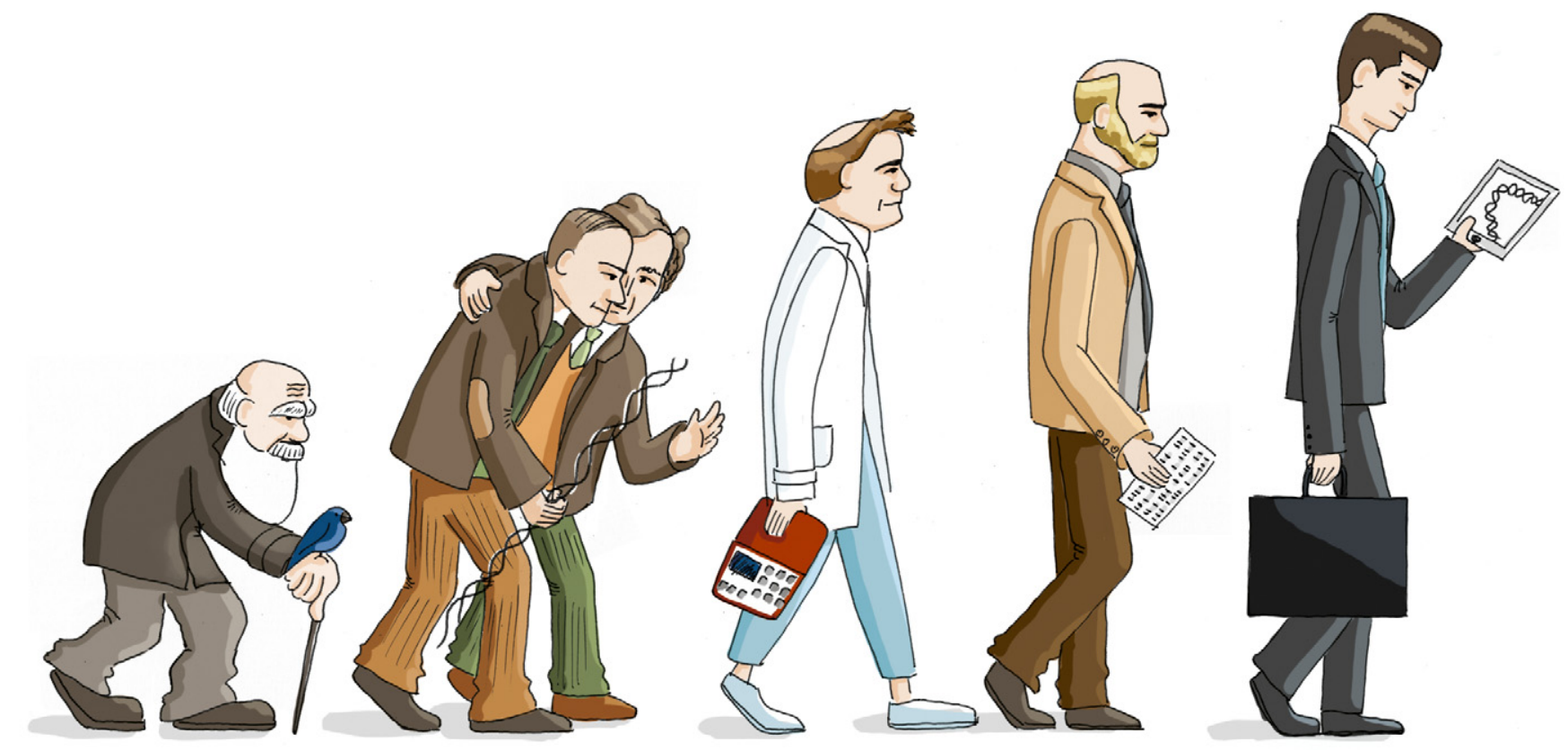

get them to plan your experiments as well. Commercial sequencing firms and other private enterprises offer comprehensive, step-by-step consulting and project-design services, and some will even address and manage aspects of clinical trials. As claimed by SeqWright, "The steadily increasing costs and regulatory requirements associated with Phase I - Phase IV Clinical Trials make it imperative that project strategies address all critical aspects of the Trial. During the last decade, many ... have found that outsourcing procedures is an efficient and reliable solution to their clinical trial requirements" (www.seqwright.com).

$\mathrm{N}$ ew technologies and the burgeoning biotech industry have made it quick, cheap, and easy for researchers to generate massive amounts of molecular sequence information [4], but they have come at a cost. A lot of scientists are struggling and scrambling to make sense of these data and consequently are contracting out their bioinformatics tasks to experts. A week barely goes by that I do not receive a barrage of emails from companies advertising bioinformatics services. This morning it was AccuraScience offering me a "one-stop solution to next-generation sequencing (NGS) data processing, analysis, and interpretation" (www.accurascience.com). A cursory scan of their website reveals an impressive array of services, such as de novo genome and transcriptome assemblies, metagenomics and methylation analyses, and ChIP-Seq interpretation-you name it, they'll do it. Other bioinformatics businesses specialize in specific areas. LC Sciences (www.lcsciences.com), for example, markets itself as a guru of RNA-Seq and transcriptome data, whereas Zymo Research (www.zymoresearch.com) is all about epigenetic analyses, and Applied Biomics (www.appliedbiomics.com) are the proteome people. As the bioinformatics trade expands, companies are going to greater and greater extremes to attract customers, offering customizable computer pipelines and servers, tailored statistical analyses, publication-quality figure and table construction, even data interpretation $[1,5]$.

What does this mean for the future of academic research? One of the many dangers of the commercialization and the farming out of science is that investigators and students will lose touch with the techniques used to generate their data and the organisms or systems from which the data are derived-a problem that I have encountered myself [6]. Great insights and discoveries often come from years of one-on-one involvement with the species being investigated as well as from sustained, first-hand experience with the experiments being carried out. Arguably, Nobel Laureate Barbara McClintock would never have had the same insights into corn genetics and transposable elements had she outsourced her crop, cytological, and molecular work to someone else. Conversely, Watson and Crick did not (and likely could not) carry out the experiments that ultimately helped them elucidate the structure of DNA - those of Rosalind Franklin.

ike it or not, the current trend toward outsourcing science is not going to change anytime soon, which may not be a bad thing for some overworked and stressed out academics. In the past, much of the energy of academic research labs went into planning and running experiments, maintaining and updating equipment and reagents, and organizing the lab. Given the resources that are available today, it is possible for researchers to outsource a large number of these tasks and devote more energy to interpreting the data, testing hypotheses, and forging new theories, which for most scientists is the best part of the job. It also means that lab groups from small colleges will be able to punch above their weight and compete with those from large, research-intensive institutes. University researchers are often restricted in what they can accomplish by the equipment and facilities available to them on campus. If the growth of scientific outsourcing continues, soon all academics, for a fee, will have access to cutting-edge equipment and advanced technologies. 
I study the genomes of algae, particularly those that have lost photosynthetic capabilities. It is now at the point where I can perform primary research without ever having to interact with the species or nucleic acids that I am investigating. For example, I could select an algal strain of interest from the NCMA culture collection, have the NCMA grow the organism, extract DNA, and then send the DNA to a commercial facility of my choosing for high-throughput sequencing, followed by professional bioinformatics consulting and analysis, catered to my project needs. At the end of this chain, I should receive polished genomic data, which I could then use to address specific questions within my area of study. All of this without touching a pipette or Eppendorf tube, without mining anyone else's data, and possibly without spending significantly more money that it would have cost me had I done the brunt of the work in-house. I have yet to try this, but would not be opposed to it.

\section{"If new ways of doing science limit the freedom of researchers ... then this will likely stifle their creativity as well."}

When I talk to my colleagues, I get the sense that they feel more like CEOs of small businesses rather than primary investigators. They spend most of their time applying for and allocating grant money, attracting topnotch personnel, fostering and balancing collaborations, and managing the input and output of scientific data from the lab. Rarely, if ever, do they spend time at the bench. But even more surprising is that I am meeting more and more postdocs and graduate students who express similar sentiments. If this is the future of research, maybe we should be encouraging science students to get an MBA, or at least provide them with some sort of managerial or business training. In fact, the university where I work just started a Master's in Management of Science
Program (www.uwo.ca/professionalmasters/ science/), as have many others. No doubt the most important thing will be teaching young scientists and students to use the resources that are available to them creatively and intelligently.

\section{“ ... if scientists stop being creative, or worse, try to contract out creativity, then it will surely mark the end of the academy."}

$\mathrm{n}$ this brave new world of outsourcing, how can we ensure that life scientists continue to think creatively about biological systems? Creativity and freedom are finely interwoven. If new ways of doing science limit the freedom of researchersthe freedom to ask the questions that they believe are important and to carry out the experiments and analyses needed to address those questions - then this will likely stifle their creativity as well. One can only hope that the commercialization of science will provide investigators with more channels and greater opportunities to accomplish their goals and test hypotheses. However, if scientists stop being creative, or worse, try to contract out creativity, then it will surely mark the end of the academy.

Moving forward, educators and PIs will need to develop new strategies to cultivate in their students an appreciation and thorough understanding of biological systems. This may require adding mandatory field and laboratory components to computer- and management-based science programs, to ensure that students remain engaged with the organisms, environments, and biological topics they are studying. I recently read about the upbringings and early educations of celebrated evolutionary biologists Bill Hamilton and Richard Dawkins. It was telling to learn how early and sustained interactions with Nature instilled a passion for biology in both of these great thinkers. No matter how technological, streamlined, and automated science becomes, investigators must avoid becoming data-generating, publication-writing machines and not lose sight of why they are a trying to understand the natural world-for many, because they want to preserve it.

When I asked one of the longest-serving members of my department how he feels about the changing scientific landscape he frowned and said: "It's very hard for me not to be reactionary when contemplating the prospect of the CEO scientist, but it will probably be the way of the future, at least for a while."

\section{Acknowledgements}

I thank Marc-André Lachance for helpful feedback on an early draft of the article. DRS is supported by a Discovery Grant from the Natural Sciences and Engineering Research Council (NSERC) of Canada. He can be found online at www.arrogantgenome.com and@arrogantgenome.

\section{Conflict of interest}

The author declares that he has no conflict of interest.

\section{References}

1. Pichler FB, Turner SJ (2007) The power and pitfalls of outsourcing. Nat Biotechnol 11 1093-1096

2. Koboldt DC, Steinberg KM, Larson DE, Wilson RK, Mardis ER (2013) The next-generation sequencing revolution and its impact on genomics. Cell 155: 27-38

3. Smith DR (2014) Last-gen nostalgia: a lighthearted rant and reflection on genome sequencing culture. Front Genet 5: 146

4. Metzker ML (2010) Sequencing technologiesthe next generation. Nat Rev Genet 11: $31-46$

5. Dick S, Hasson U (2010) Outsourcing neuroimaging data analysis: implications for scientific accountability and issues in the public interest. Trends Cogn Sci 14: 2-4

6. Smith DR (2013) Are we divorced from the species we study? F1000Res 2: 254 